Biorheology Vo1. 15, p. 141

(C) Pergamon Press Ltd. 1978. Printed in Great Britain

ANNOUNCEMENTS

BRITISH MICROCIRCULATION SOCIETY

FUTURE MEETINGS

12th World Conference of the European Society for Microcirculation 23-28 October 1978, Forte Village, S. Margherita di Pula, Cagliari, Sardinia

Abstract should be sent to the President - Professor G. Brotzu, Insituto di Chirurgia Toracica de11'Universita di Cagliari, Ospedale - R. Binaghi, Monteurpinu, Cagliari, Italy. No positive information about costs, accommodation etc. has yet been sent to me, but it is understood that the conference fee will be $\$ 112$ U.S. if paid before 30 April or $\$ 120$ thereafter but $\$ 20$ addition if not a member of the European Society.

American Microcirculation Society - 2nd World Conference 22-29 July 1979, at La Jolla, California, U.S.A.

\title{
COMBINED MEETING
}

XII International Conference on Medical and Biological Engineering and V International Conference on Medical Physics, 19-24 August, 1979, Jerusalem, Israel.

For information, please contact the Conference Secretariat, F.0. Box 16271, Te1 Aviv, Israel.

\section{ERRATUM}

The publisher wishes to apologize for the following errors: In the paper by P. F. Dunn and B. F. Picologlu, in Biorheology 14, pp. 277-292.

1. Equation (8): $\lambda$ should bé $\gamma$.

2. Equation (15): $Q(T)$ should be $Q(t)$.

3. Equation (17): ( $(\partial u / \partial r) e$ should be $(\partial u / \partial r)_{e}$.

4. Expression in text between equations (20) and (21): $\beta_{b}$ should be $\beta^{b}$.

5. Ref. 19: In press should be changed to 22, 217 (1977). 